\title{
Low 25-OH vitamin D level are associated with increased postoperative acute kidney injury, broch pleural fistula after lung transplantation
}

\author{
Nam Eun Kim ${ }^{1}$, Ha Eun $\mathrm{Kim}^{2}$, Song Yee Kim, Beom Seok Kim ${ }^{3}$, Jung Tak Park ${ }^{3}$, Jin Gu Lee ${ }^{2}$, Hyo Chae Paik ${ }^{3}$, Moo Suk Park
}

\footnotetext{
${ }^{1}$ Division of Pulmonology, Department of Internal Medicine, Severance Hospital, Seoul, Korea

${ }^{2}$ Department of Thoracic and Cardiovascular Surgery, Severance Hospital, Seoul, Korea

${ }^{3}$ Division of Nephrology, Department of Internal Medicine, Severance Hospital, Seoul, Korea
}

Background: Vitamin D (Vit D) deficiency is found in over two-thirds of lung transplantation (LT) candidates, which is greater than in general population. In addition to bone metabolism, low serum level of 25-hydroxyvitamin $\mathrm{D}[25(\mathrm{OH}) \mathrm{D}]$ is known to be associated with higher incidence of infections, cancer, and cardiovascular disease. So, it is important to investigate association between Vit D level and post LT complications.

Methods: In this single-center study, we reviewed 237 LT patients from January 2013 to July 2019 at Severance Hospital, Seoul, Korea. Thirty-nine patients were excluded due to missed level of preoperative Vit D. Enrolled patients were divided into Vit D deficiency group (range, $0-10 \mathrm{ng} / \mathrm{mL}$ ), Vit $D$ insufficiency (range, $10-30 \mathrm{ng} / \mathrm{mL}$ ), and Vit D sufficiency group $(>30 \mathrm{ng} / \mathrm{mL}$ ). Analysis of variance and chi-square tests were used for comparing variables.

Results: Forty-nine patients (24.7\%) were Vit D deficiency, 128 patients (64.6\%) were Vit D insufficiency, and 21 patients (10.6\%) were Vit D sufficiency. There were no significant differences between three groups with respect to age, sex, comorbidities, and primary lung disease. Low Vit D level was associated higher occurrence of postoperative acute kidney injury (AKI; deficiency vs. insufficiency vs. sufficiency, 10 [22.2] vs. 11 [9.5] vs. 1 [5.0]; $P<0.05)$. Prevalence of broncho pleura fistula was higher in Vit $D$ sufficiency group: deficiency vs. insufficiency vs. sufficiency, $0(0.0)$ vs. $14(10.9)$ vs. $0(0.0)$; $P=0.016$. We further evaluate postoperative broch pleural fistula (BPF) in Vit $D$ increase and Vit $D$ decrease group within 6-month follow-up, and Vit $D$ decrease group show high prevalence of BPF: decrease vs. increase, 9 (12.3) vs. 1 (1.1); $P=0.002$. Low Vit $D$ level was also associated with postoperative anxiety, but it was not statically meaning.

Conclusions: Low 25-OH Vit D level is associated with increased incidence of post LT complications such as AKI, BPF, and anxiety. Also, Vit D decrease during postoperative period showed higher incidence of BPF.

Corresponding author: Moo Suk Park

E-mail:PMS70@yuhs.ac

(C) The Korean Society for Transplantation

This is an Open Access article distributed under the terms of the Creative Commons Attribution Non-Commercial License (http://creativecommons.org/licenses/by-nc/4.0/) which permits unrestricted non-commercial use, distribution, and reproduction in any medium, provided the original work is properly cited. 